\title{
Size control, quantum confinement, and oxidation kinetics of silicon nanocrystals synthesized at a high rate by expanding thermal plasma
}

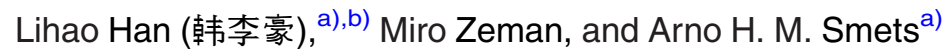 \\ Photovoltaic Materials and Devices (PVMD) Laboratory, Delft University of Technology, P.O. Box 5031, \\ 2600 GA Delft, The Netherlands
}

(Received 28 February 2015; accepted 15 May 2015; published online 27 May 2015)

\begin{abstract}
The growth mechanism of silicon nanocrystals ( $\mathrm{Si} \mathrm{NCs}$ ) synthesized at a high rate by means of expanding thermal plasma chemical vapor deposition technique are studied in this letter. A bimodal Gaussian size distribution is revealed from the high-resolution transmission electron microscopy images, and routes to reduce the unwanted large Si NCs are discussed. Photoluminescence and Raman spectroscopies are employed to study the size-dependent quantum confinement effect, from which the average diameters of the small Si NCs are determined. The surface oxidation kinetics of Si NCs are studied using Fourier transform infrared spectroscopy and the importance of postdeposition passivation treatments of hydrogenated crystalline silicon surfaces are demonstrated. (C) 2015 AIP Publishing LLC. [http://dx.doi.org/10.1063/1.4921760]
\end{abstract}

Ensembles of nanocrystals (NCs) have attracted intensive attention in research due to their various special properties. NCs can be considered as zero-dimensional nano-structures whose excitons are confined in all three spatial dimensions. They have the ability to tune the band gap by varying their sizes and have unique interactions with photons like the multiple exciton generation (MEG) ${ }^{1}$ or up-/down-spectral conversion by space separated quantum cutting (SSQC). ${ }^{2}$ These unique mechanisms make NCs promising novel applications in thin-film transistors (TFTs), ${ }^{3}$ water-splitting devices, ${ }^{4}$ batteries, ${ }^{5}$ and solar cells. ${ }^{6,7}$ For instance, the utilization of MEG or SSQC properties of NCs might open new routes to conquer the Shockley-Queisser limit of single-junction solar cells, ${ }^{8}$ if the Shockley-Read-Hall charge carrier recombination at the surfaces can be tackled. In 2011, above $100 \%$ external quantum-efficiency (EQE) value in the blue spectral range has been achieved in a cell using PbSe NCs. ${ }^{9}$

Herein we focus on NCs made of silicon ( $\mathrm{Si}$ ), which is non-toxic, earth abundant, resistance against water, and consequently known as the most dominant material in semiconductor and photovoltaic (PV) industry. ${ }^{6,10-14}$ Si NCs are generally fabricated by sputtering, ${ }^{15}$ pulsed laser deposition, ${ }^{6}$ or non-thermal plasma. ${ }^{16,17}$ Each process has its specific disadvantages, such as, limited production rate, complex and time-costly processes, post-annealing treatment, poor control over the size distribution, and low degree of surface passivation. The slow deposition rate, low throughput, and complex synthesis procedures limit the large-scale application of $\mathrm{Si}$ NCs. In this work, expanding thermal plasma chemical vapor deposition (ETP-CVD) technique is employed to synthesis $\mathrm{Si}$ NCs in a super-efficient rate. ETP-CVD technique was initially developed at the TU/Eindhoven for the high-rate deposition of thin-film materials. ${ }^{18-20}$ Pioneering work was done for the deposition of $\mathrm{Si}$ NCs as well. ${ }^{21,22}$ However, some key fundamental growth mechanisms and properties of

\footnotetext{
a) Authors to whom correspondence should be addressed. Electronic addresses: hanlihao@gmail.com and A.H.M.Smets@tudelft.nl.

${ }^{b}$ Current affiliation: Laboratory of Renewable Energy Science and Engineering (LRESE), École Polytechnique Fédérale de Lausanne (EPFL), 1015 Lausanne, Switzerland.
}

Si NCs processed by ETP-CVD have not been explored yet, such as the oxidation kinetics and the size-dependent photoluminescence (PL) mechanism of the Si NCs. In this letter, we further explore the growth mechanisms of ETP-CVD to synthesize Si NCs under various deposition conditions, deposit free-standing Si NCs with a well-controlled size distribution for any specific application, and investigate the quantum confinement effects dependent on the size of $\mathrm{Si}$ $\mathrm{NCs}^{23,24}$ and the oxidation kinetics at the surfaces of Si NCs.

The photo of the ETP-CVD setup and typical deposition parameters for the synthesis of $\mathrm{Si} \mathrm{NCs}$ are shown elsewhere. ${ }^{21,22,25}$ The morphology of Si NCs is characterized by scanning electron microscopy (SEM) and high resolution transmission electron microscopy (HR-TEM), and the images with different magnifications were shown in Figs. 1(a)-1(c). The Si nano-power was deposited in the form of a porous film as observed in the top-view SEM image (Fig. 1(a)). HRTEM was operated at a voltage of $200 \mathrm{kV}$ to analyze the sizes of NCs at larger magnifications, and clear lattice fringes can be observed if Fig. 1(b) is zoomed in. Two typical sizes of $\mathrm{Si}$ NCs are discovered: NCs with diameters in the range of $2-8 \mathrm{~nm}$ (4 $\mathrm{nm}$ in average, $\sim 1600 \mathrm{Si}$ atoms, defined as "small" in this letter, Fig. 1(b)) and NCs with diameters of 20-38 nm (28 $\mathrm{nm}$ in average, defined as "large" in this letter, Fig. 1(c)). The size of deposited NCs is mainly determined by the residence time and subsequently the trajectory of Si species in the plasma beam from the injection ring down to the substrate. The small NCs were collected on the substrate spots which were exactly below the holes on the $\mathrm{SiH}_{4}$ injection ring. Therefore, we believe that these small NCs were formed in the plasma beam and directly deposited on the substrate. Its trajectory through the plasma is a straight vertical line from the injection ring down to the substrate, resulting in a short residence time in the plasma. In contrast, the large NCs correspond to particles that have travelled through the background of the chamber before they were deposited on the other areas on the substrate. They have a larger average diameter as a result of longer residence time and trajectory in the plasma chamber due to the recycling from the background volume into the plasma beam. ${ }^{21}$ Therefore, the size of NCs is 


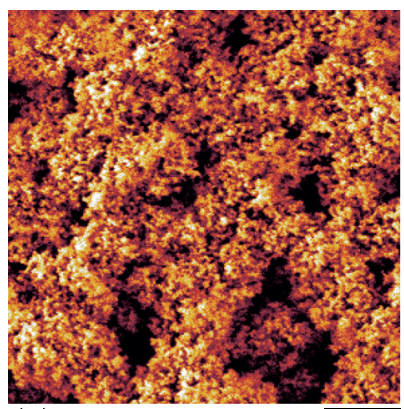

(a)

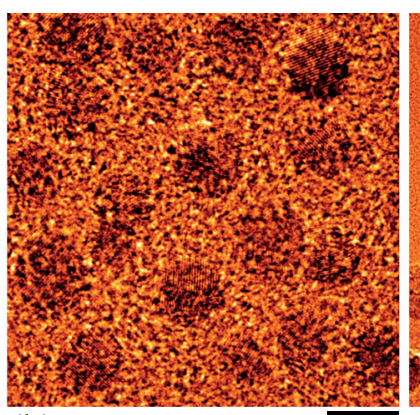

(b)
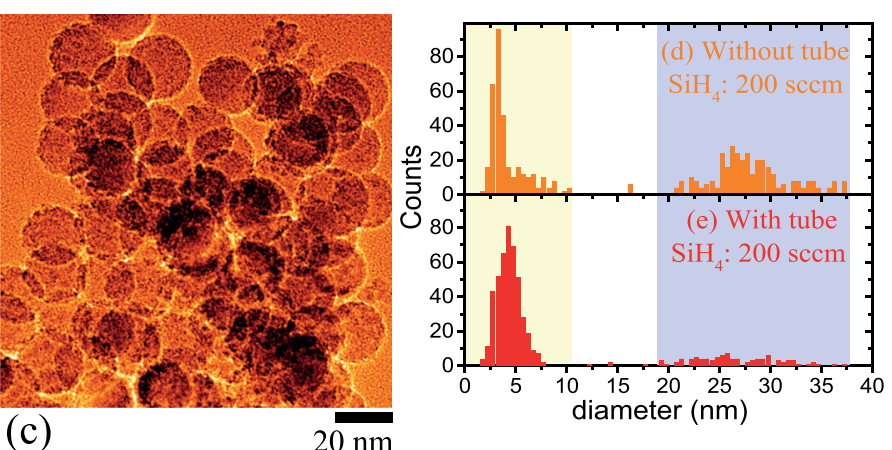

FIG. 1. Top-view SEM image of the ensembles of Si NCs (a), and the HR-TEM images of the small (b) and large (c) Si NCs. The size distribution analysis of the Si NCs from HR-TEM images deposited by the initial ETP-CVD without a tube (d) and by the modified ETP-CVD with a tube to reduce the diameter of the reaction chamber (e).

mainly determined by the residence time of Si species in the plasma beam from the injection ring down to the substrate, and subsequently depends on parameters such as pressure, gas flow, and plasma power. The statistics of bimodal size distribution based on HR-TEM images is analyzed in Fig. 1(d), and it is a result of NCs deposited directly from the plasma beam and recycling of NCs from the background volume into the plasma beam. The small NCs exhibit quantum confinement effects, while the large ones do not. Therefore, various solutions have been proposed to minimize the travelling time of the silyl radicals in the plasma beam from the injection holes to the substrate, such as by rising the substrate stage or descending the injection ring for a shorter traveling distance of the silyl radicals, injecting the $\mathrm{SiH}_{4}$ precursor in a pulsed mode, ${ }^{21}$ or reducing the diameter of the reaction chamber. Fig. 1(e) shows an example of reducing the reaction chamber by installing a tube around the ring. The tube significantly reduces the amount of Si NCs that follows the path of the recycling trajectory. Consequently, the contribution of large NCs in the films can be greatly reduced or the formation of large NCs can even fully quenched.

To further study the growth mechanism of Si NCs, we varied the $\mathrm{SiH}_{4}$ gas flow rate for three samples of the $\mathrm{Si}$ NCs to 230,420 , and $600 \mathrm{sccm}$, respectively, while keeping all the other deposition parameters the same. Figs. 2(a)-2(c) show the size distribution analysis of these three ensembles of $\mathrm{Si}$ NCs collected on other spots (not below the holes of the $\mathrm{SiH}_{4}$ injection rings) on the substrates. The average sizes are $27 \pm 6,35 \pm 16$, and $41 \pm 19 \mathrm{~nm}$, respectively, for the large $\mathrm{Si}$ NCs of these three samples. It is interesting to see that the average diameter of these large free-standing NCs increase with the $\mathrm{SiH}_{4}$ gas flow, as shown in Fig. 2(d) (black left-axis). This can be understood in a simple model in which the increase of the volume $V$ of the particles in time is linear with the surface area $\left(A=\pi d^{2}\right)$ and growth flux $\phi: \mathrm{d} V \sim \pi d^{2} \phi \mathrm{d} t$. As a result, the diameter of the particle is linear with the flux and residence time $\tau_{\text {res }}: d \sim \phi \tau_{\text {res }}$. The result in Fig. 2(d) shows that the average diameter of the Si NCs $d$ is roughly linear with the silane flux $\phi$, which is a reasonable assumption.

The blue symbols in Fig. 2(d) (right-axis) show the deposition rate of Si NCs, which were collected from a piece of CORNING glass substrate with an area of $10 \mathrm{~cm} \times 10 \mathrm{~cm}$. The $\mathrm{SiH}_{4}$ gas flow was varied step by step, while the other deposition parameters were kept the same. This rate was determined by the weight gain of the substrate before and after deposition. It can be seen that the deposition rate increases with the $\mathrm{SiH}_{4}$ gas flow and the highest rate of $1.9 \mathrm{mg} \mathrm{cm}^{-2} \mathrm{~min}^{-1}$ was achieved when the $\mathrm{SiH}_{4}$ gas flow was kept at $600 \mathrm{sccm}$. This rate is several orders higher than those deposited by other techniques such as sputtering, ${ }^{15,26}$ pulsed laser deposition, ${ }^{6}$ or non-thermal plasma. ${ }^{16,17}$ The ETP-CVD is so efficient that several micrometers of the Si NCs porous layer can be deposited on the substrate in $1 \mathrm{~s}$. The silane utilization efficiency is high as estimated from the slope of the blue line in Fig. 2(d). We find that $\sim 40 \%$ of $\mathrm{Si}$ atoms in the injected silane were collected on the $10 \mathrm{~cm} \times 10 \mathrm{~cm}$ substrate in the form of $\mathrm{Si}$ nano-powder. Besides the high deposition rates, ETP-CVD synthesis has the advantages of deposition at room temperature, low cost, high purity, and integration of post-deposition surface passivation treatments using state-ofthe-art vacuum processing technologies.

The size-dependent quantum confinement of the small $\mathrm{Si}$ NCs was studied by PL and Raman spectroscopy. Therefore, the Si NCs were deposited on glass substrate using the tube-
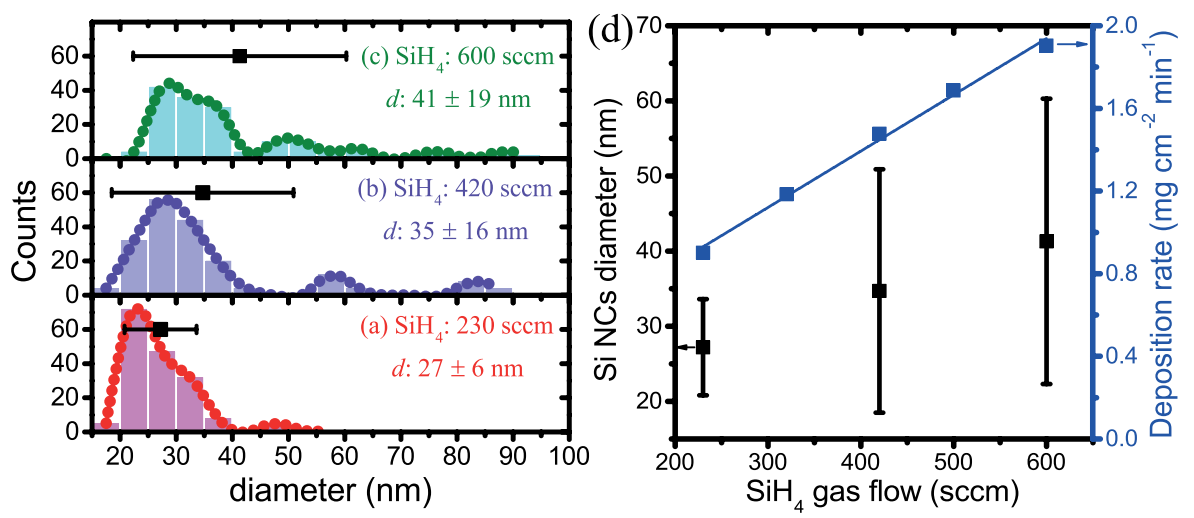

FIG. 2. The size distribution of three samples of Si NCs deposited by 230, 420, and $600 \mathrm{sccm}$ of $\mathrm{SiH}_{4}$ precursors ((a)-(c)), the average diameters of the large Si NCs exhibit an increasing trend with the $\mathrm{SiH}_{4}$ gas flow ((d), black left-axis), and the high deposition rate of Si NCs in the series of the injected $\mathrm{SiH}_{4}$ gas flow ((d), blue right-axis). 
configuration. Three samples of the ensembles of small Si NCs were collected in the spots on the substrates below the holes of the $\mathrm{SiH}_{4}$ injection rings, and they were deposited by varying the $\mathrm{SiH}_{4}$ gas flow rate $(230,420$, and $600 \mathrm{sccm}$, respectively). The samples were immediately placed into the PL setup after the deposition to prevent any effects of post-deposition oxidation of the surfaces of the Si NCs. In the PL setup, the NCs were illuminated by a laser source with the excitation wavelength of $420 \mathrm{~nm}$. The emission PL signals were detected at the peak positions of 810,830 , and $880 \mathrm{~nm}$ for these three samples, respectively, as shown by the normalized spectra in Fig. 3(a). According to literature, ${ }^{27-29}$ the average diameters of the small Si NCs can be estimated as 3.5, 4.0, and $4.5 \mathrm{~nm}$ for the three samples (corresponds to $\mathrm{SiH}_{4}$ gas flow rate 230, 420, and $600 \mathrm{sccm}$ ). It is estimated from the PL emission peak positions that the optical bandgaps of the small Si NCs in these three samples are $1.40,1.49$, and $1.53 \mathrm{eV}$, respectively, which are in between those of crystalline $\mathrm{Si}(\mathrm{c}-\mathrm{Si}, 1.1 \mathrm{eV})$ and hydrogenated amorphous $\mathrm{Si}(\mathrm{a}-\mathrm{Si}: \mathrm{H}, 1.8 \mathrm{eV})$.

The quantum confinement effects of the as-deposited small NCs were further characterized using Raman spectroscopy under Argon laser illumination $(514 \mathrm{~nm})$ at a low intensity $(3.3 \mu \mathrm{W})$. It should be pointed out that the laser intensity should be as mild as possible in order to prevent the heating effect of the free-standing NCs that naturally have a poor thermal conductivity. ${ }^{30}$ The spectra of three as-deposited samples are shown in Fig. 3(b). The first-order Si-Si mode Stokes peak shift of Si NCs in the Raman spectrum is lower by a few wavenumbers in reference to the number observed for bulk c-Si $\omega_{0}=520.5 \mathrm{~cm}^{-1}$, as observed by many others. ${ }^{6,31-34}$ In general, this observation is attributed to phonon confinement, due to the presence of small NCs embedded in the a-Si:H environment. Assuming that this shift can be fully attributed to quantum confinement effect, the average small NCs size can be estimated from the anharmonic Raman softening according to this formula ${ }^{35}$

$$
d=2 \pi\left(\frac{\mathrm{C}}{\Delta \omega}\right)^{\frac{1}{2}} .
$$

Here, $d(\mathrm{~nm})$ stands for the diameter of the average NCs size, and $\mathrm{C}=2.24 \mathrm{~nm}^{2} \mathrm{~cm}^{-1}$, which is a constant for Si material. $\Delta \omega$ is the absolute wavenumber $\left(\mathrm{cm}^{-1}\right)$ difference between $\omega_{0}$ and the measured $\omega$ for $\mathrm{Si}$ NCs, i.e.,
$\Delta \omega=\left|\omega_{0}-\omega\right|$. The average diameters of the Si NCs can be determined from Eq. (1). Assuming that quantum confinement effect is indeed responsible for this red-shift, we find an average size for the small NCs of 4.0, 5.0, and $5.9 \mathrm{~nm}$, respectively, for the three samples processed with increasing $\mathrm{SiH}_{4}$ gas flow $(230,420$, and $600 \mathrm{sccm})$. These results are quite close to the sizes determined by PL emission peak positions, in spite of a small overestimation due to the fact that the Raman technique is more sensitive to the larger NCs in reference to PL. The relevance of the $\Delta \omega$ and PL peak position is presented in Fig. 3(c).

The Shockley-Read-Hall recombination at defect-rich surfaces or interfaces is one of the key issues that limits the application of Si NCs in highly efficient solar cells or other electronic devices. The passivation of the surface of Si NCs is therefore crucial. Under ambient atmospheric conditions, a native oxide layer is formed on the surfaces of the Si NC, which acts as a poor passivation layer. In order to better understand the post-deposition oxidation, we employ Fourier transform infrared (FTIR) transmission spectroscopy and monitor the sample of Si NCs for one-week exposure in the air. The surface oxidation kinetics of Si NCs without any post-deposition surface-passivation treatment is studied and presented in Fig. 4(a). Besides the typical stretching modes for hydrogenated $\mathrm{Si}$ surfaces $\left(\mathrm{Si}_{4-\mathrm{y}}-\mathrm{Si}-\mathrm{H}_{\mathrm{y}} @ \sim 2106 \mathrm{~cm}^{-1}\right),{ }^{36}$ various stretching modes can be observed that represent bonding configurations that include oxygen as well. The majority of the oxygen gets incorporated in a $\mathrm{Si}-\mathrm{Si}$ bond as shown by the Si-O-Si stretching modes (1050, 1107, and $\left.1160 \mathrm{~cm}^{-1}\right){ }^{36-38}$ This is also shown in the $\mathrm{Si}-\mathrm{H}$ stretching modes with at least one oxygen atom back-bonded to the $\mathrm{Si}$ atom $\left(\mathrm{OSi}_{2}-\mathrm{Si}-\mathrm{H} @ 2140 \mathrm{~cm}^{-1}, \mathrm{O}_{2} \mathrm{Si}-\mathrm{Si}-\mathrm{H} @ 2190 \mathrm{~cm}^{-1}\right.$, and $\mathrm{O}_{3}-\mathrm{Si}-\mathrm{H} @ 2250 \mathrm{~cm}^{-1}$ ). This demonstrates that most of the oxygen is incorporated in the back bonds of the $\mathrm{Si}$ atoms at the surface of Si NCs. In addition, the bending mode of the $\mathrm{O}_{3}-\mathrm{Si}-\mathrm{H}$ configuration has been observed at $881 \mathrm{~cm}^{-1}$ as well. The measurement discloses that the pure $\mathrm{Si}$ hydrides $\left(\mathrm{Si}_{4-\mathrm{y}}-\mathrm{Si}-\mathrm{H}_{\mathrm{y}}\right)$ bonds at surfaces almost disappeared after one day as a result of the exposure to air as shown in Fig. 4(b) and the black curve in Fig. 4(c)). On the other hand, the configurations that have an oxygen incorporated in the Si back bond increase on the same time scale. This shows that the hydrogenated Si surfaces oxidized rapidly. After a fast oxidation within the first day of air exposure, the oxidation
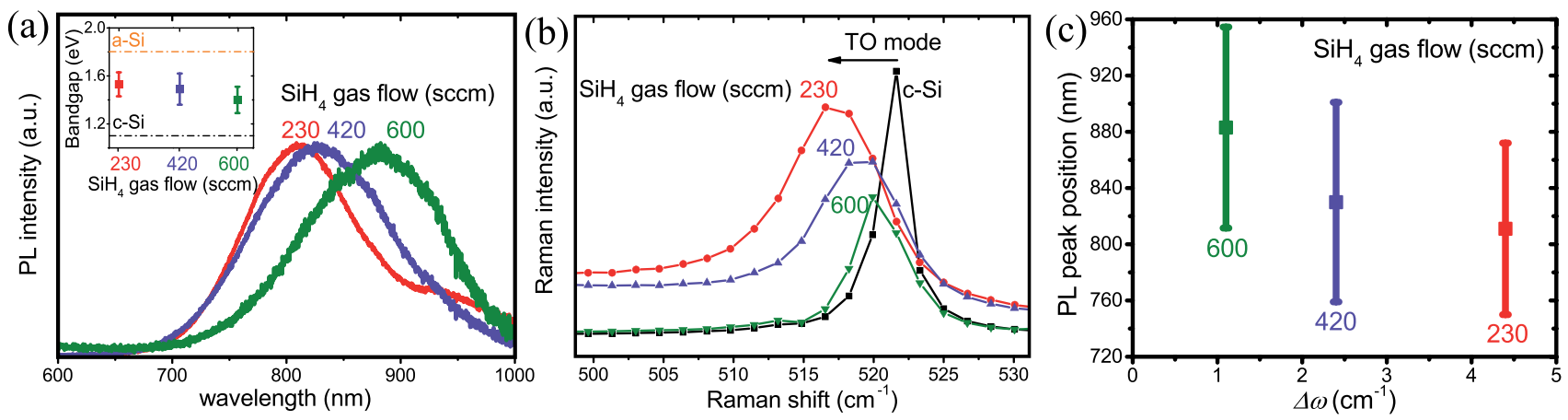

FIG. 3. Photoluminescence spectra of the small Si NCs in the series of $\mathrm{SiH}_{4}$ gas flow (a), and the optical bandgaps of the small Si NCs estimated from the PL emission peaks are in between those of crystalline $\mathrm{Si}$ and amorphous $\mathrm{Si}$ (inset of (a)). Raman spectra of the small $\mathrm{Si} \mathrm{NCs}_{\mathrm{in}}$ the series of $\mathrm{SiH} \mathrm{H}_{4}$ gas flow (b), and the relevance of the Raman shift of the Si-Si TO mode peak with the PL peak positions (c). The vertical bars indicate the full width at half maximum (FWHM) of each PL peak. 

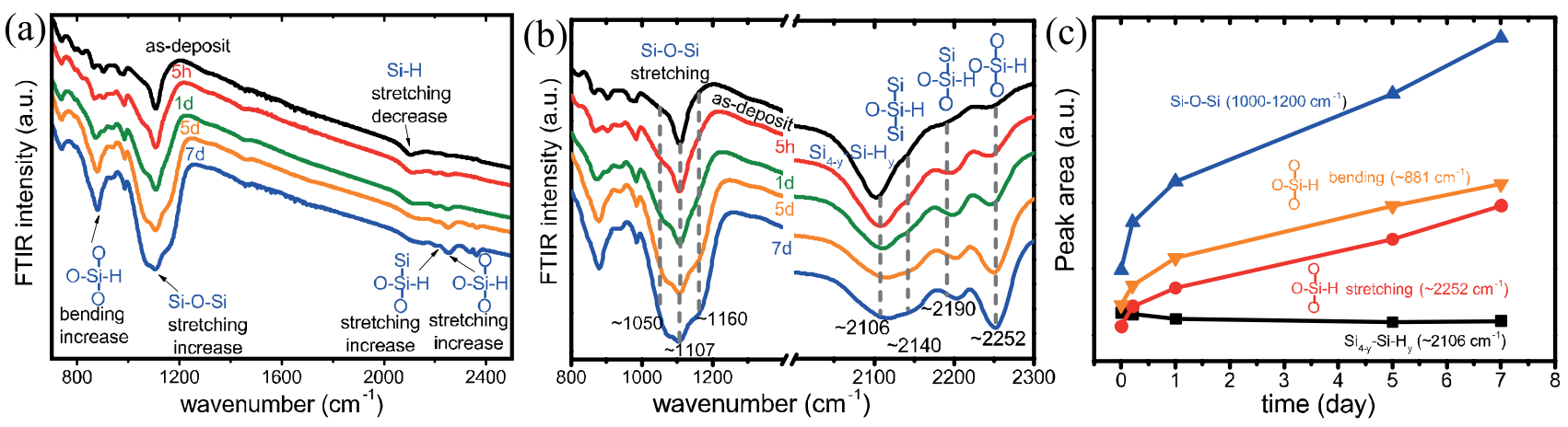

FIG. 4. FTIR monitoring during one week after the deposition of Si NCs (a), and the zoomed-in spectra in the interesting ranges (b). Peak area analysis shows the incorporation of oxygen in the back bonds of the silicon hydrides increasing in the first week after deposition (c).

continued, but at a lower rate. The oxidization was not saturated yet after 7 days in air. The increase of the Si-O-Si stretching modes, the $\mathrm{O}_{\mathrm{x}}-\mathrm{Si}-\mathrm{H}$ stretching modes, and bending modes has a similar trend, which means that the Si-O-Si stretching modes correspond to $\mathrm{Si}-\mathrm{O}-\mathrm{Si}$ bonds close to the surface of $\mathrm{Si}$ NCs. The observed oxidation significantly affects the chemical properties of the surface of Si NCs. This demonstrates the importance of post-deposition passivation treatment of hydrogenated crystalline $\mathrm{Si}$ surfaces when the $\mathrm{Si}$ NCs are applied in devices.

In summary, the deposition mechanism of Si NCs by means of ETP-CVD technique has been demonstrated in this letter. ETP-CVD was verified as an efficient process for the fabrication of $\mathrm{Si} \mathrm{NCs}$ on an industrial scale. A bimodal Gaussian size distribution was revealed by analyzing the HR-TEM images, and routes to reduce the amount of the unwanted large Si NCs were discussed. PL and Raman spectroscopies were employed to study the size-dependent quantum confinement effect, and the average diameters of the small Si NCs determined by these two methods were similar. The FTIR results showed the surface oxidation kinetics at the surfaces of Si NCs and demonstrated the importance of post-deposition passivation treatments of hydrogenated crystalline silicon surfaces.

Financial support from the VIDI Project granted to Dr. A. H. M. Smets by NWO-STW is gratefully acknowledged. The authors thank our colleagues Martijn Tijssen and İlker Doğan for the discussions on processing.

${ }^{1}$ T. Trupke, M. A. Green, and P. Wurfel, J. Appl. Phys. 92(3), 1668 (2002). ${ }^{2}$ D. Timmerman, I. Izeddin, P. Stallinga, I. N. Yassievich, and T. Gregorkiewicz, Nat. Photonics 2(2), 105 (2008).

${ }^{3}$ F. Hetsch, N. Zhao, S. V. Kershaw, and A. L. Rogach, Mater. Today 16(9), 312 (2013).

${ }^{4}$ Y. Tachibana, L. Vayssieres, and J. R. Durrant, Nat. Photonics 6(8), 511 (2012).

${ }^{5}$ Y. Kwon, G. S. Park, and J. H. Cho, Electrochim. Acta 52(14), 4663 (2007). ${ }^{6} \mathrm{~L}$. Han, J. Wang, and R. Liang, IEEE Photovoltaics Specialists Conference (IEEE, 2010), p. 3338.

${ }^{7}$ L. Han, J. Wang, and R. Liang, Adv. Mater. Res (Durnten-Zurich, Switz.) 383-390, 6270 (2012).

${ }^{8}$ W. Shockley and H. J. Queisser, J. Appl. Phys. 32(3), 510 (1961).

${ }^{9}$ O. E. Semonin, J. M. Luther, S. Choi, H. Y. Chen, J. B. Gao, A. J. Nozik, and M. C. Beard, Science 334(6062), 1530 (2011).

${ }^{10}$ F. F. Abdi, L. Han, A. H. M. Smets, M. Zeman, B. Dam, and R. van de Krol, Nat. Commun. 4, 2195-1 (2013).

${ }^{11}$ L. Han, F. F. Abdi, P. Perez Rodriguez, B. Dam, R. van de Krol, M. Zeman, and A. H. M. Smets, Phys. Chem. Chem. Phys. 16(9), 4220 (2014).
${ }^{12}$ L. Han, F. F. Abdi, R. van de Krol, R. Liu, Z. Huang, H.-J. Lewerenz, B. Dam, M. Zeman, and A. H. M. Smets, ChemSusChem 7(10), 2832 (2014).

${ }^{13}$ L. Han, I. A. Digdaya, T. W. F. Buijs, F. F. Abdi, Z. Huang, R. Liu, B. Dam, M. Zeman, W. A. Smith, and A. H. M. Smets, J. Mater. Chem. A 3, 4155 (2015).

${ }^{14}$ L. Han, F. F. Abdi, R. van de Krol, B. Dam, M. Zeman, and A. H. M. Smets, in 40th IEEE Photovoltaic Specialist Conference (IEEE, 2014), p. 3083.

${ }^{15}$ S. J. Huang and G. Conibeer, J. Phys. D: Appl. Phys. 46(2), 024003 (2013).

${ }^{16}$ R. Gresback, T. Nozaki, and K. Okazaki, Nanotechnology 22(30), 305605 (2011).

${ }^{17}$ O. Yasar-Inceoglu, T. Lopez, E. Farshihagro, and L. Mangolini, Nanotechnology 23(25), 255604 (2012).

${ }^{18}$ R. Groenen, J. Loffler, P. M. Sommeling, J. L. Linden, E. A. G. Hamers, R. E. I. Schropp, and M. C. M. van de Sanden, Thin Solid Films 392(2), 226 (2001).

${ }^{19}$ K. Sharma, B. L. Williams, A. Mittal, H. C. M. Knoops, B. J. Kniknie, N. J. Bakker, W. M. M. Kessels, R. E. I. Schropp, and M. Creatore, Int. J. Photoenergy 2014, 253140.

${ }^{20}$ M. C. M. van de Sanden, R. J. Severens, W. M. M. Kessels, R. F. G. Meulenbroeks, and D. C. Schram, J. Appl. Phys. 85(2), 1243 (1999).

${ }^{21}$ I. Dogan, N. J. Kramer, R. H. J. Westermann, K. Dohnalova, A. H. M. Smets, M. A. Verheijen, T. Gregorkiewicz, and M. C. M. van de Sanden, J. Appl. Phys. 113(13), 134306-1 (2013).

${ }^{22}$ I. Dogan and M. C. M. van de Sanden, J. Appl. Phys. 114(13), 134310-1 (2013).

${ }^{23}$ N. M. Park, C. J. Choi, T. Y. Seong, and S. J. Park, Phys. Rev. Lett. 86(7), 1355 (2001).

${ }^{24}$ T. Takagahara and K. Takeda, Phys. Rev. B 46(23), 15578 (1992).

${ }^{25}$ L. Han, Ph.D. thesis, Delft University of Technology, 2015.

${ }^{26}$ S. Park, E. Cho, D. Y. Song, G. Conibeer, and M. A. Green, Sol. Energy Mater. Sol. Cells 93(6-7), 684 (2009).

${ }^{27}$ W. D. A. M. de Boer, D. Timmerman, K. Dohnalova, I. N. Yassievich, H. Zhang, W. J. Buma, and T. Gregorkiewicz, Nat. Nanotechnol. 5(12), 878 (2010).

${ }^{28}$ G. Ledoux, O. Guillois, D. Porterat, C. Reynaud, F. Huisken, B. Kohn, and V. Paillard, Phys. Rev. B 62(23), 15942 (2000).

${ }^{29}$ C. M. Hessel, D. Reid, M. G. Panthani, M. R. Rasch, B. W. Goodfellow, J. W. Wei, H. Fujii, V. Akhavan, and B. A. Korgel, Chem. Mater. 24(2), 393 (2012).

${ }^{30}$ L. Han, M. Zeman, and A. H. M. Smets, Nanoscale 7, 8389 (2015).

${ }^{31}$ G. Conibeer, M. Green, E. C. Cho, D. Konig, Y. H. Cho, T. Fangsuwannarak, G. Scardera, E. Pink, Y. D. Huang, T. Puzzer, S. J. Huang, D. Y. Song, C. Flynn, S. Park, X. J. Hao, and D. Mansfield, Thin Solid Films 516(20), 6748 (2008).

${ }^{32}$ S. K. Gupta and P. K. Jha, Solid State Commun. 149(45-46), 1989 (2009).

${ }^{33}$ L. Sirleto, M. A. Ferrara, T. Nikitin, S. Novikov, and L. Khriachtchev, Nat. Commun. 3, 1220-1 (2012).

${ }^{34}$ Z. F. Sui, P. P. Leong, I. P. Herman, G. S. Higashi, and H. Temkin, Appl. Phys. Lett. 60(17), 2086 (1992).

${ }^{35}$ G. X. Cheng, K. J. Chen, W. Zhang, H. Xia, and X. K. Zhang, Phys. Status Solidi A 129(2), 421 (1992).

${ }^{36}$ X. D. Pi, L. Mangolini, S. A. Campbell, and U. Kortshagen, Phys. Rev. B 75(8), 085423 (2007).

${ }^{37}$ A. C. Bronneberg, A. H. M. Smets, M. Creatore, and M. C. M. van de Sanden, J. Non-Cryst. Solids 357(3), 884 (2011).

${ }^{38}$ X. D. Pi and U. Kortshagen, Nanotechnology 20(29), 295602 (2009). 